\title{
AC 2010-1388: INTEGRATION OF MODERN ENERGY STORAGE DESIGN PRACTICES INTO UNIVERSITY AUTOMOTIVE ENGINEERING PROGRAMS
}

Frank Falcone, Argonne National Laboratory

Terrence Davidovits, A123 Systems

Eric Schacht, The Ohio State Univeristy

Michael Wahlstrom, Argonne National Laboratory 


\title{
Title of the Paper: Integration of Modern Energy Storage Design Practices into University Automotive Engineering Programs
}

\begin{abstract}
As automotive technology surges forward at an ever increasing pace, Argonne National Laboratory works to keep the US Department of Energy sponsored Advanced Vehicle Technology Competitions current by introducing university students to cutting edge tools, technologies, and design practices. One major addition to the AVTCs is energy storage system design. Traditionally, AVTC energy storage systems were purchased components which students integrated into vehicles. However, with advanced vehicles like the Chevrolet Volt nearly to market, the AVTC steering committee found that off the shelf energy storage solutions were no longer adequate to enable students to sufficiently push efficiency and performance envelopes. The competition's scope has been expanded to enable teams to design custom energy storage solutions using cutting edge Lithium Iron Phosphate battery technology and design processes.

AVTC organizers worked with the competition's primary energy storage sponsor, A123 Systems, to develop the logistical and technical infrastructure to define schedules, deliverables, training, technical support, and provide hardware to the teams. Students worked through the summer of 2009 preparing comprehensive design reports which were evaluated by a team of industry subject matter experts from A123 systems, Argonne National Laboratory and General Motors. The design process brought computational fluid dynamics, dimensional analysis, thermodynamics, cooling system design, control strategy development, failure identification and mitigation, and finite element analysis up to new levels within the competition. Passage of these technical evaluations was necessary to receive energy storage hardware. These engineering experiences help the AVTC continue to cultivate the talent required to support modern automotive research, development, and design.
\end{abstract}

\section{Introduction to the AVTC}

Over the span of twenty two years, the Advanced Vehicle Technology Program managed by Argonne National Laboratory and sponsored by the US Department of Energy as strived to bring government, industry and academia together to meet timely automotive related challenges in an educational environment. Primarily, these challenges have been powertrain centric focused on reducing energy consumption and tail pipe emissions. The engineering work is performed by multiple disciplines of college level engineering students thereby exposing students to the types of problems they will need to solve if they chose to work in the automotive industry. In the competition's early years, most of the engineering was performed by mechanical engineering students. Students modified engines to run on various alternative fuels such as propane, methanol, and ethanol. As technology evolved, so did the competitions as hybrid powertrain architectures entered the field. This expanded the required engineering skill sets to include electrical and electro-mechanical engineering experience to the program. In 2004, Challenge X began which expanded the competition series time frame from 1 year to 3. Argonne, the US DOE, and General Motors worked to design a program that integrated a modified vehicle development process into the academic environment. This program further tasked students with 
more advanced control system development and design thus bringing yet another engineering discipline to the table.

In 2008, EcoCAR the NeXt Challenge kicked off with headline sponsors General Motors and the US DOE working with Argonne to integrate the latest in automotive engineering technology in the collegiate environment. The EcoCAR competition consists of the following 17 universities:

\author{
Missouri Science \& Technology \\ Texas Tech \\ West Virginia University \\ Georgia Tech \\ Rose-Hulman Institute of Technology \\ Penn State \\ University of Wisconsin Madison \\ North Carolina State University \\ University of Ontario Institute of Technology \\ Michigan Tech \\ Howard University \\ The Ohio State University \\ University of Waterloo \\ University of Victoria \\ Mississippi State University \\ Virginia Tech \\ Embry-Riddle Aeronautical University
}

The AVTC's role in curricula development is typically one an initiator. The competitions bring new challenges to teams and supply hardware while coordinating training and deliverables to assist the students. Universities then have the opportunity to absorb these materials and add to them as they custom tailor their programs to support advanced vehicle design.

In EcoCAR, control system development became a primary focus of the first year and a half. The advent of plug-in hybrid technology in combination with more advanced lithium ion energy storage created a new set of demands and challenges to the universities because the vehicles became increasingly electrified. It is here where Argonne and A123 systems collaborated to 
design the educational structure, training, and engineering deliverables to teach and guide EcoCAR students in the practice of modern energy storage design, something that had never been formally addressed in the competition environment before.

\section{Introduction to the Vehicles of EcoCAR, The NeXt Challenge}

EcoCAR has a diverse fleet of advanced vehicle technologies which adds to the challenge of evaluating each ESS design. The technologies are grouped as follows. Fuel cell plug-in hybrid electric vehicles (FCPHEV's) are vehicles powered by hydrogen fuel cells but have significant battery powered driving capability which means they can be recharged with electricity each day to lessen hydrogen consumption. Full function electric vehicles (FFEV's) are vehicles with very large ESS and power themselves only with electricity supplied by their ESS. The Hybrid Electric Vehicle (HEV) architectures use bio diesel or ethanol engines of varying sizes in combination with an ESS that is not recharged from a source outside the vehicle. Plug-In Hybrid Electric Vehicles (PHEV's) in general require large battery packs similar to what is found in the FCPHEV's storing significant energy for all-electric driving range delivering high power to driving demands. HEV's while requiring less storage capacity still require batteries that can accept and deliver power at a high rate. FCHEV's require the storage capacity of a typical PHEV but have the added issues of less mass and packaging volume headroom which can make integration of cooling more difficult. FFEV's require their electric powertrains to meet all driving conditions at any given time over a large range. The results of these powertrain characteristics are ESS's that are very different from one another but all requiring careful scrutiny during the design review process.

To add more real world constraints to the design process, the vehicle test criteria are set to capture the vehicle in multiple driving conditions. There are performance events such as acceleration and handling that test the peak powertrain performance. The towing even tests the continuous power delivery capabilities. Fuel consumption and emissions tests the overall system design from a control and integration standpoint since things like mass and poor powertrain calibration can have adverse affects on fuel consumption and resulting emissions. Drive quality is assessed through objective and subjective tests in multiple operational modes to further evaluation how well the whole system is designed and controlled. The overall EcoCAR design goal or philosophy is one of balance where the efficiency is increased, emission reduced, while production performance characteristics are maintained or exceeded.

\section{Energy Storage System Design Challenges}

Energy Storage System (ESS) design confronts the competition engineering students in ways that other powertrain integration does not. For example, electrical engineering students design wiring schematics that denote wire size, fusing, and other circuit protection. These students perform circuit design tasks such as printed circuit board development and noise suppression. Before, much of the pre-designed components were "black boxed" or not modifiable by the student engineers. The parts had recommended cable sizes with circuit protect and condition built in. Traditionally competition tasks mechanical engineering (ME) students with designing mounting brackets and sometimes power transmission assemblies. In ESS design, mounting strategies are more complex. Students must analyze multiple failure points to ensure the system is safe in the 
event of a crash. All points of failure must withstand high loading in all three axes. They must also analyze systems to predict vibration characteristics and then design mitigation strategies that conform to the mounting strength requirements. Before EcoCAR, engine vibrations might be unpleasant, but they did not make the vehicle inherently unreliable. Now vibrations can wear down and damage expensive ESS components. Off-the-shelf ESS typically have built in vibration damping.

Another ME student challenge not often faced in an AVTC is thermal cooling system design. Previously, integrated components would either come with the cooling hardware, or have specifications which made purchasing of required hardware rather straight forward. The specifications must now be defined by the students and would not take place if the students did not design their own ESS.

ESS design challenges teams to manage the collaboration required to accomplish the design. Control and simulation students gain more experience through their collaboration with their fellow ME team members. Proposed cooling system designs are brought into the rapid controls prototyping process where simulations help the students develop control solutions for the ESS they have designed. These help to define and scope their powertrain operational modes. The modes help students understand loading and cooling requirements. ME students then perform thermodynamic analysis to help guide their design solution. Typically with a pre-designed ESS, these limits are published and there is no trade off to be considered. The operational limits are hard set. Now, students can weight vehicle performance against, for example, efficiency losses of operation more powerful cooling pumps or the added mass of larger cooling systems.

\section{Case Study: OSU's AVTC Program}

While it requires significant time and support from organizers and sponsors such as A123 to accomplish this work, it almost always requires even more from the students themselves. One prime example of successful university ESS design practices is that done by the engineering students at The Ohio State University in Columbus Ohio.

The Ohio State University (OSU) has participated in AVTC events for nearly two decades, which makes them one of the longest running universities in the program. In these two decades, the university has created an entire Center for Automotive Research (CAR) that houses not only industry and private research, but also many student vehicle competitions, relating to automotive and transportation research and technology in a variety of ways. The AVTC teams are not the only OSU student teams to work with large ESS designs previously the formula lightning program was responsible for an all electric formula car and now the team has become the buckeye bullet land speed program, which shares a design interest in ESS like those used in the AVTC vehicles. The OSU research and student teams continue to find many ways to apply and learn about ESS design and implementation for the automotive sector.

During this progression and participation OSU's knowledge and success with ESS has grown and improved. In prior vehicles to that of EcoCAR, OSU has chosen batteries with considerably less energy ( $<2 \mathrm{kWhr}$ compared to as much as $22 \mathrm{kWh}$ ). However, during the EcoCAR design process OSU determined that not only was the industry moving quickly towards high power and 
energy packs, but that consumers were demanding it and interested in all electric vehicle operation. The desire to satisfy consumer desires and push the technology boundary led the team to design a vehicle with considerable electric range and all electric performance. Batteries on the market were barely capable of achieving this goal and none of the available products came in an appropriate pack. A123 offered support far beyond other battery companies and also has a superior product able to greatly improve the vehicle performance. The collaboration with A123 allowed a customizable battery pack from a set of modules, which allowed the team freedom to integrate the batteries into a more ideal system for the vehicle design. Therefore, it will be seen how the collaboration worked with A123 and Argonne National Laboratory and has provided the team with a large part of their winning design.

\section{ESS Design in EcoCAR}

As mentioned, the EcoCAR ESS design process required that teams perform design work in several different disciplinary categories. To help guide students, a structure was created to train students so that they could meet deliverables at a preset schedule. The main deliverable to earn ESS components was a comprehensive design report.

To effectively train students in the process of ESS design, two methods were employed, workshops and tech talks. Each competition year students are flown to a common destination where competition organizers and sponsor give training sessions at a live workshop. Typically, there is one workshop in the fall at or near the beginning of the academic year and one in winter (January) half way through the academic year. At the first fall workshops, for example, A123 Systems defined and gave sessions that introduced teams to their products and the design process onto which the students would embark. To divulge more technical information large conference calls, or tech talks, were set up where students could view a live presentation on their computer screens while asking questions real time. Sponsors such as A123 gave more hands on training at the winter workshop. This process continued for the first one and a half years of the competition.

As stated previously, the main deliverable was a comprehensive ESS design report. To simplify the report generation and design evaluation process, categories were broken down broadly into Electrical, Mechanical, Thermal and Safety with each area being reviewed by subject matter experts. Despite this breakdown into disciplinary elements, the most successful teams during the EcoCAR ESS design process were those that applied a system oriented or "Top Down" design process. Teams that struggled often did not define the system and its requirements first instead going straight to component design. Therefore, the process was setup such that it reinforced the system level design approach teams are required to use when selecting their powertrains and applied it to solving the complex technical challenges here.

The first step was to determine what size ESS would be needed to optimize vehicle performance in line with competition and team goals. Teams were allowed to assemble custom packs out of two different varieties of prismatic battery modules, each with a different voltage and energy. This optimization process was not explicitly reviewed by A123 and left entirely up to the individual team's discretion. Next, modeling was conducted using the MATLAB/Simulink based vehicle modeling software Powertrain Systems Analysis Toolkit (PSAT). Some assumptions about vehicle hybrid drivetrain control logic had to be made at this junction. Teams were 
required to summarize the conclusions drawn from their PSAT modeling and provide a high level explanation of their control methodology. A key output from the PSAT modeling process was the power levels the team wanted to operate their battery at. With power levels and battery capacity requirements determined, teams could proceed to detailed design of the battery pack in the disciplinary areas: Thermal, Electrical, Mechanical and Safety.

In order to be approved to receive A123 prismatic battery modules, teams had to conduct an analysis of their cooling requirements using a combination of battery specifications provided by A123 and loading characteristics from their respective powertrains. These cooling requirements were dependent on the current each module needed to provide - small HEV packs faced a greater challenge than large PHEV packs due to their reduced thermal mass, heavy regenerative braking and propulsion power demands increasing cooling demand. Teams were able to pursue either air cooled or liquid cooled solutions, with most teams opting for liquid cooled "over-ambient" solutions (i.e. without the use of chilled fluid). For these liquid cooled solutions, the bulk of the work consisted of designing a cold plate, often in conjunction with computational fluid dynamics packages (CFD), and selecting appropriate radiators and pumps to accommodate the cold plates. Then the operation of these elements in conjunction with the battery had to be quantitatively modeled to obtain the temperature rise in each component, including the battery module itself, and thus determine the "hot spot" in the cells. Analyses varied widely in the level of sophistication between teams, with some teams employing significant amounts of CFD, running transient simulations, or accounting for the temperature dependence of heat generation in the battery cells and other teams sticking to simple hand calculations. The level of complexity of analysis and the capability of the thermal system was up to the teams to balance and prioritize; approval hinged on correctly putting together the analysis and demonstrating an understanding of how the system would function in ambient conditions the team's had chosen to design to. For the mechanical design, teams were tasked with efficiently packaging their battery modules and electronic components into the car. Most teams targeted the rear cargo area for this purpose, with teams having larger packs facing significantly more of a challenge than teams with smaller packs. The bulk of the work then went into designing a pack housing that could withstand the gforces that might be encountered in the event of a collision - $20 \mathrm{~g}$ in the forwards, backwards and lateral directions and $8 \mathrm{~g}$ up and down. The vast majority of teams employed FEA for this purpose. Each structural element in the potential failure chain from module to vehicle frame needed to be assessed. Each team also needed to perform a simple vibration analysis to ensure a sufficiently high natural frequency for the battery pack. Further, teams needed to develop a cell venting strategy, generally by way of a check valve that would open in the event several cells should fail and vent gas into the battery pack. The valve would allow the evacuation of this gas outside the vehicle.

\section{Case Study: OSU's ESS Design}

The OSU EcoCAR design process involved the typical OEM structure, which first defines an obtainable set of vehicle technical specifications (VTS). In the case of OSU the most unique VTS goal was an all electric range and a significant reduction in fuel usage. This challenged the vehicle design in two ways. First the vehicle had to have a large electric Powertrain, and second the vehicle needed a large ESS. Each by itself did not present a major difficulty in design, however, combined it risked increased weight and difficult packaging. 
The team began with an ESS design using cells, which provided a very versatile pack with custom made cooling and packaging. The pack was at first cost prohibitive and then with the support of A123 the first pack was abandoned for more energy dense battery modules. Therefore, the team began designing packaging, cooling, and controls for the ESS that was to be sponsored by A123. This design process eventually showed three stages. Preliminary design concepts and discussion, then revised design and analysis, lastly the final approved design and implementation plan. During these three stages it was expected that the team provide a complete design, but as feedback was given and the design was discussed it was shown how the design lacked details important to proving safety, serviceability, and constructability. Therefore, the ESS design showed that it was no longer possible to just use a component and integrate it without computer aided design. It was now necessary to design on a much broader level and much more intense level using a variety of analysis tools.

In past competition vehicles, one to three people might have been directly responsible for the ESS system, which provided direct accountability, but also provided safety with few persons fully immersed in the servicing and contact with the ESS. In the OSU EcoCAR design, the ESS design process included 5-7 students and multiple advisors this required training, documentation, communication, file version management, and timelines. The design process has prompted additional safety training, initial file storage training, and file naming conventions. When training new members it is re-iterated how parts will be manufactured and how they will be serviced in order to keep these items in the design.

To meet these needs, OSU has to take a look at ways to properly structure their team. In the past the OSU team has specialized by major or experience to accomplish tasks in mechanical design, electrical design, and software design. These specializations would accomplish tasks quickly, but interact poorly and often lack synergy in overall design implementation. Both time management and physical implementation issues were presented. The specialized team structure did not allow room for expansion due to difficulty in working in parallel if for example all electrical members showed up the tools and equipment had to be shared among the group, and in design there were no clear boundaries or interdisciplinary checks and balances. Therefore the team structure was modified to include all relevant disciplines for the tasks, but divide the groups and work based on subsystems. This provided better communication and better synergy in completing the design of a subsystem such as the ESS. For example the ESS previously would have been the responsibility of mostly electrical team members and partially the controls or software team members. In the EcoCAR ESS design the ESS was set as a subsystem, which included mechanical packaging design, electrical wiring design, and controls management and fault diagnosis. This collaboration helps to create a cohesive and unified design.

\section{Lessons Learned}

With many new processes one comes away with ideas of how to improve things for the next time and this is no different. From the organizer perspective, it is important to reflect on the system level design principles employed in all other areas of the competition and place them into context in this new frontier of collegiate automotive design. It was realized during the past year that some teams did not have a firm understanding of the "big picture" issue before delving into the 
details. A degree of freedom was given to the students as far as how they went about solving their design which meant in some cases students had to rework their design following top down principles they did not initially employ. Refining the deliverable structure to force teams through a top down design process could expedite the overall design approval.

Workload impacts on scheduling were not well understood at first. Firm due dates and clear methods of submission help students manage time and resources while helping organizers to evaluate and return the work quickly. In EcoCAR, deliverable ambiguity caused due dates to be adjusted which meant final approval deadlines slipped. Future competitions will refine what was done in EcoCAR so that the deliverable has a clearer template to increase the quality and organization of deliverables while maintaining proper scope to help expedite the review process. Definition of engineering skill sets at the onset could prove valuable to faculty and staff as they develop and evolve their EcoCAR teams. Heat transfer concepts often proved problematic. Front loading initial competition training with these needs can give faculty time to adjust classes or bring in other faculty experts to help guide students.

From the university's perspective, front loading training can improve the end product. Spending time upfront on training for more students, especially younger students, provides many more people to input ideas and work on designs. Making a team structure that allows design in parallel to be interdisciplinary ensures more checks and balances on all design aspects. In addition, interdisciplinary design brings more creative solutions to the design. Providing access to information and datasheets upfront helps improve involvement. Brainstorming and discussion with sponsors or industry experts should be done before a design report is made using simple drawings or models to offer talking points. This discussion and brainstorming provides an opportunity to ask questions more freely before a design is even attempted. In designs such as the ESS many different stages take time and therefore a student given only senior year or one or two semesters or quarters to complete such a design might miss learning an entire part of the project. For example the mechanical packaging must be done before final wiring design can be done, therefore a student that leaves after only the conclusion of the packaging would never learn how to integrate the wiring properly. In addition, this early completion of projects for students leaves them without experience on servicing or assembling the system. The serviceability and therefore, assembly of an ESS is very critical to safety and reliability, therefore, if the student is not forced to do assembly or service on the system they do not understand the design shortcomings. Students should be started in academia on projects and design in conjunction with their academics rather than to conclude their academics. Implementing this philosophy has helped enrich OSU student educational experience while producing a better final product.

\section{Conclusion}

The EcoCAR ESS design process encountered some difficulties throughout the first year and a half of the three year competition series. However, the process has gelled and appears to be a great success. The program is educating student engineers to levels previously unforeseen in competitions largely in part due to the incorporation of ESS design. The vehicle performance is expected to rise accordingly. To keep the competition and its graduates ahead of the automotive design curve, it is expected that ESS design will now be a staple for the foreseeable future. In the AVTC's this is and will continue to be accomplished through collaboration between 
government, industry and academia.

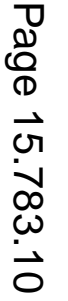

